\title{
Opfer des Seebebens in Südostasien: Unfall oder Krankheit?
}

\section{J. Petermann Büttler}

Das am 26. Dezember 2004 erfolgte Seebeben in Südostasien, welches zu einer der grössten bekannten Flutkatastrophen geführt hat, hat die Verletzlichkeit der Menschen und ihrer kulturellen Leistungen (wieder einmal) sehr deutlich gemacht. Ebenso deutlich zeigte sich eine Welle der Solidarität, die um die ganze Welt ging. Auch Schweizerinnen und Schweizer haben geholfen, direkt vor Ort oder von hier aus mit Geldspenden. Auch Behörden und Ämter haben schnell reagiert und unbürokratische Verfahren versprochen.

Am 12. Januar 2005 haben sich auf Initiative von santésuisse Vertreter des BAG, BSV, EDA, SVV und der Suva getroffen, um sich im Zusammenhang mit dem verheerenden Seebeben, das auch viele Schweizer Bürgerinnen und Bürger getroffen hat, in versicherungsrechtlichen Belangen auszutauschen und ein gemeinsames Verständnis des sogenannten Schreckereignisses zu schaffen. Da die Versicherungsleistungen nach einem Unfall gemäss Unfallversicherungsgesetz (UVG) und Krankenversicherungsgesetz (KVG) unterschiedlich sind, ist es für eine versicherte Person unter Umständen von grosser Bedeutung, wer für ihre Versehrtheit aufkommt. Das KVG macht für seine Versicherten keinen Unterschied zwischen krank oder verunfallt. Wichtig ist deshalb auch, dass die Ärztinnen und Ärzte, welche vom Seebeben betroffene Personen behandeln, die seelische Verletzungen davongetragen haben, in Versicherungsfragen richtig beraten können.

\section{Verletzt - aber nicht körperlich}

* Vgl. dazu die Rechtsprechung gemäss BGE 129 V 177; U 46/04.

Korrespondenz:

Dr. iur. Judith Petermann Büttler santésuisse

Ressort Recht

Römerstrasse 20

CH-4500 Solothurn

E-Mail:

judith.petermann@santesuisse.ch
Grundsätzlich kann bei von der Flutwelle unmittelbar betroffenen Personen von einem Schreckereignis ausgegangen werden. Denn Rechtsprechung und Lehre* haben bei dramatischen und heftigen Ereignissen, die eine Störung des psychischen Gleichgewichts bewirken, unter bestimmten Voraussetzungen den Unfallbegriff bejaht und hierfür bestimmte Kriterien aufgestellt.
Nachfolgend erlauben wir uns, Beispiele aufzuführen, bei denen Personen im Zusammenhang mit der Flutkatastrophe in Ostasien psychische Beschwerden entwickeln könnten, ohne dass sie selber körperlich verletzt worden sind. Es handelt sich dabei insbesondere um folgende Möglichkeiten:

1. Personen, die sich in Todesgefahr befanden und trotzdem vor der Flutwelle retten konnten;

2. Personen, welche die Flutwelle direkt erlebt haben, die aber aus objektiver Sicht nicht in Todesgefahr standen;

3. Personen, welche zwar die Flutwelle nicht selbst erlebt haben, die aber in den betroffenen Gebieten mit den direkten Auswirkungen der Flutwelle (Zerstörung, Verstorbene, Rettungsdienste usw.) konfrontiert worden sind, sei dies als Touristen oder als Helfer;

4. Angehörige von Verletzten, Vermissten und Verstorbenen;

5. Personen, welche über die Auswirkungen der Flutwelle via Medien oder Erlebnisberichten informiert worden sind.

\section{Der Einzelfall zählt}

Bei den Personen gemäss den Punkten 3 bis 5 entspricht das Ereignis nicht der EVG-Definition eines Unfalls, weil sich der Vorfall (die Flutwelle oder das Beben) nicht in unmittelbarer Gegenwart der entsprechenden Personen ereignet hat. Somit gelten alle Behandlungen dieser Personen als Behandlungen infolge Krankheit und unterliegen dem KVG.

Hingegen wird man bei den Personen unter Punkt 1, welche wegen der Flutwelle in unmittelbarer Todesgefahr standen und sich glücklicherweise retten konnten, davon ausgehen, dass das entsprechende Schreckereignis grundsätzlich geeignet ist, zumindest vorübergehende psychische Beschwerden hervorzurufen. Entsprechende Behandlungen gelten deshalb als Behandlungen infolge eines Unfalls. Schwieriger zu beantworten ist bei diesen Fällen die Frage, ob 
und allenfalls ab welchem Zeitpunkt die psychischen Folgen nicht mehr in einem adäquaten Kausalzusammenhang mit der Flutkatastrophe stehen. Jeder Einzelfall wird individuell zu beurteilen sein.

Versicherungstechnisch besonders schwierig wird die Beurteilung der Personen gemäss Punkt 2 sein. Jeder Einzelfall wird speziell gewürdigt werden müssen. Die Personen, welche subjektiv in der konkreten Situation für sich eine Todesgefahr annehmen mussten, sind eher den Personen gemäss Punkt 1 gleichzustellen.

\section{Triage durch Ärztinnen und Ärzte}

Diese dargelegten Differenzierungen können den behandelnden Ärztinnen und Ärzten Anhaltspunkte geben, ob sie dem Patienten via Arbeitgeber zu einer Unfallmeldung an ihre Unfallversicherung raten sollen. Selbstverständlich muss letztlich in jedem Einzelfall unter Würdigung aller Umstände abgeklärt werden, ob ein Unfall vorliegt. Dabei handelt es sich um eine Rechtsfrage. Für die versicherte Person jedoch, welche zum Beispiel eine hohe KVG-Wahlfranchise abgeschlossen hat, ist es unter Umständen von eminenter Bedeutung, ob ihr Unfallversicherer die Leistungen ausrichtet oder ihre Krankenversicherung.

\title{
Victimes du tsunami en Asie du sud-est: maladie ou accident?
}

\author{
J. Petermann Büttler
}

* Voir à cet égard la jurisprudence selon ATF 129 V 177; U 46/04.

Correspondance:

Dr iur. Judith Petermann Büttler santésuisse

Ressort Droit

Römerstrasse 20

CH-4500 Soleure

E-mail:

judith.petermann@santesuisse.ch
Le tsunami qui s'est produit en Asie du sud-est le 26 décembre 2004 et qui a provoqué l'un des plus grands raz-de-marée de tous les temps, a très clairement révélé (une fois de plus) la précarité de la condition humaine et la fragilité des infrastructures créées par l'homme. Mais il a aussi déclenché une chaîne de solidarité internationale. Des Suissesses et des Suisses sont également venus en aide aux sinistrés, sur place, ou depuis notre pays, par des dons en espèces. Même les autorités et les administrations ont réagi rapidement et efficacement.

La vague dévastatrice a aussi fait de nombreuses victimes parmi nos compatriotes. C'est pourquoi des représentants de l'OFSP, de l'OFAS, du DFAE, de l'ASA et de la Suva se sont réunis le 12 janvier 2005 à l'initiative de santésuisse afin de se concerter sur un certain nombre de questions d'assurance et de développer une compréhension commune des conséquences de l'évènement traumatisant. En effet, les prestations d'assurance après un accident diffèrent selon que la loi sur l'assurance accidents (LAA) ou la loi sur l'assurance-maladie (LAMal) sont applicables. Il est donc capital pour les patients de savoir quel assureur prendra leurs frais de guérison en charge. La LAMal ne fait aucune différence entre la maladie et l'accident. Il importe donc que les médecins, qui soignent des victimes du tsunami souffrant d'atteintes psychologiques, les conseillent de manière avisée.

\section{Des blessures à l'âme}

On peut partir du principe que les personnes directement touchées par la catastrophe ont subi un choc émotionnel. La jurisprudence et la doctrine* reconnaissent la notion d'accident dans certaines conditions, notamment lors d'événements dramatiques majeurs entraînant un déséquilibre psychique, et ont défini des critères correspondants.

Nous vous indiquons ci-après quelques exemples de situations dans lesquelles des personnes pourraient développer des troubles psychiques suite à la catastrophe en Asie du sud-est sans avoir été blessées physiquement, notamment:

1. les personnes qui ont été en danger de mort, mais qui ont pu échapper au raz-de-marée;

2. les personnes qui ont directement assisté au raz-de-marée, mais qui n'ont objectivement pas été en danger de mort; 
3. les personnes qui n'ont pas vécu directement le raz-de-marée, mais qui ont été confrontées dans les régions touchées aux conséquences directes de la catastrophe (destruction, morts, service de secours, etc.), en qualité de touriste ou sauveteur;

4. les proches de personnes blessées, disparues ou défuntes;

5. les personnes qui ont été informées des conséquences du raz-de-marée par les médias ou par l'intermédiaire de témoignages.

\section{Evaluation au cas par cas}

Les personnes décrites aux points 3 à 5 ne correspondent pas à la définition d'un accident selon le TFA, dans la mesure où l'événement (le raz-demarée ou le tremblement de terre) ne s'est pas produit à leur proximité immédiate. Par conséquent, tous les soins prodigués à ces personnes sont considérés comme des traitements pour maladie et relèvent donc de la LAMal.

En revanche, pour les personnes désignées au point 1 , dont la vie a été directement menacée par le raz-de-marée et qui ont pu échapper à la mort, on partira du principe que le choc subi peut provoquer des troubles psychologiques, tout du moins passagers. Les traitements administrés sont donc considérés comme des traite- ments suite à un accident. Dans ces cas-là, il sera plus difficile de dire s'il n'existe plus de lien de causalité adéquat entre les conséquences psychiques et le tsunami, et éventuellement à partir de quel moment. Chaque cas devra donc être évalué individuellement.

En terme d'assurances, la situation des personnes décrites au point 2 s'avère très délicate. Là aussi, il conviendra d'examiner minutieusement chaque cas. Les personnes qui ont été exposées subjectivement à un danger de mort dans une situation concrète doivent plutôt être assimilées aux personnes définies au point 1 .

\section{Tri par les médecins}

Les différences évoquées pourront aider les médecins traitants à décider s'ils doivent conseiller à leurs patients de faire une déclaration d'accident par l'intermédiaire de leur employeur. Il va de soi qu'il faudra en définitive déterminer au cas par cas, en tenant compte de tous les éléments, si la notion d'accident est remplie. Il s'agit là d'une question de droit. Pour les assurés qui ont, par exemple, opté pour une franchise élevée dans la LAMal, la prise en charge des prestations par leur assureur-accidents ou leur assureur-maladie revêtira une importance capitale. 